\title{
ADSORPTION OF MALACHITE GREEN AND CONGO RED DYES FROM WATER: RECENT PROGRESS AND FUTURE OUTLOOK
}

\author{
ADSORPCJA ZIELENI MALACHITOWEJ \\ I CZERWIENI KONGO Z WODY: \\ OSTATNIE POSTĘPY I PERSPEKTYWY
}

\begin{abstract}
Global concern on dyes-laden effluent has intensified over the years. Dyes are toxic, stable to light, and hardly oxidized and bio-degraded, hence causing severe physiological effects to living organisms. In water, dye hinders the light penetration for photosynthetic activity, consequently oxygen is deficient for respiration by aquatic creatures. Adsorption has been widely recognized as the effective removal strategy to abate dye wastewater. However, the quests to improve the adsorption efficiency are continuously sought through new adsorbents with special characters, while performing the removal process at optimum operating conditions. This short review aims to summarize the recent progress in adsorption studies of two commonly used industrial dyes, namely malachite green and congo red by various adsorbents. From the quoted studies, the oxidized mesoporous carbon yields a higher adsorption capacity of malachite green at $1265 \mathrm{mg} / \mathrm{g}$, while $\mathrm{Fe}_{3} \mathrm{O}_{4} @ \mathrm{nSiO}_{2} @ \mathrm{mSiO}_{2}$ displays a greater capacity for congo red removal at $1429 \mathrm{mg} / \mathrm{g}$. A superior adsorption relies not only on specific surface area but also the synergistic interactions of pore width and mesoporosity, surface chemistry, and operating conditions. The dyes properties and factors affecting the adsorption are also highlighted and discussed, with recommendations and future outlook.
\end{abstract}

Keywords: adsorbent, adsorption, congo red, malachite green, removal mechanism

\section{Introduction}

Dye is an organic compound that absorbs light in visible region. The main attributes of dye include chromogene-chromophore conjugate system (electron acceptor), resonance of electron (delocalized $\pi$-electron of aromatic ring) and solubility. Chromogene is an aromatic ring of benzene, naphthalene or anthracene, which binds chromophore (e.g., azo $(-\mathrm{N}=\mathrm{N}-)$, methine $(-\mathrm{CH}=)$, carbonyl $(=\mathrm{C}=\mathrm{O})$, etc. $)$ in double conjugated links. The conjugate system is responsible for the electromagnetic absorption that imparts colour. The presence of electron donor-ionizable functional groups known as auxochrome

\footnotetext{
${ }^{1}$ Centre of Lipids Engineering \& Applied Research (CLEAR), Ibnu-Sina Institute for Scientific \& Industrial Research, Universiti Teknologi Malaysia, 81310 UTM Johor Bahru, Johor, Malaysia

${ }^{2}$ School of Ocean Engineering, Universiti Malaysia Terengganu, 21030 Kuala Terengganu, Terengganu, Malaysia

${ }^{3}$ School of Chemical \& Energy Engineering, Universiti Teknologi Malaysia, 81310 UTM Johor Bahru, Johor, Malaysia

*Corresponding author: abbas@cheme.utm.my
} 
(e.g., amino $\left(-\mathrm{NH}_{2}\right)$, carboxyl $(-\mathrm{COOH})$, hydroxyl $(-\mathrm{OH})$, etc.) improves the solubility of dye in water. They intensify the colour and confer the binding capacity of dye onto materials. Dye is typically used for colouring products in heavy industries such as textile, paper and food processing. It is reported that the discharge of dyes in effluent accounts for $20 \%$ of water pollution [1]. Consequently, water pollution results from the release of dye in industrial effluents is hazardous and harmful to the aquatic ecosystem, food chain and public health.

Malachite green and congo red are among the commonly used industrial dyes in manufacturing industries such as textile, paper and food processing. Malachite green is a cationic dye, belonging to triphenyl methane. Its auxochrome group is protonated in water at low $\mathrm{pH}$ to bear a positive charge density $\left(\mathrm{p} K_{\mathrm{a}}=10.3\right)$ [2]. Malachite green is used for dyeing cotton, jute, paper, silk, wool and leather products. Also, it is used to treat parasites, fungal and bacterial infections in fish farming industry [3]. Despite of its wide applications, malachite green in water brings about negative effects to aquatic living creatures and human health due to its toxic characteristics. It damages liver, spleen, kidney and heart, inflicts lesions to skin, eyes, lungs and bones, and produces teratogenic effects to nervous system and brain [2,3]. Congo red is an anionic dye with $\mathrm{p} K_{\mathrm{a}}$ of 4.5 [4]. It is commonly used in textile, paper, rubber and plastic industries [5]. In water, it creates a red colloidal solution, in which it is expected to metabolize to benzidine, a substance that has been reported to be carcinogenic and mutagenic to aquatic organisms [6]. Generally, the discharge of dyes into water resources even in a small amount is aesthetically displeasing, reduces light penetration, and affects the gas solubility for photosynthesis and respiration processes. While, physical contact with dissolved dyes may result in eye and skin irritation to human [7]. Table 1 summarizes the properties of malachite green and congo red dyes.

Table 1

Properties of malachite green and congo red dyes

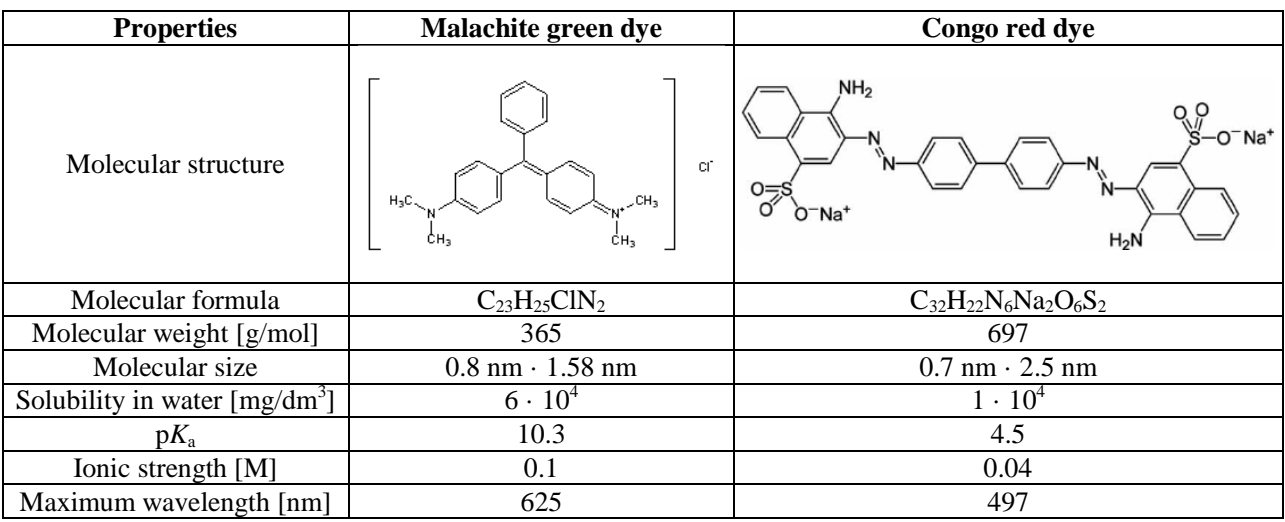

Dyes are water-soluble and exhibit inherent chemical structure that is stable to photo-degradation and bio-degradation. A number of physicochemical and biological methods have been reported for the removal of dyes from wastewater. Among others, biological treatment is preferred for pilot-scale remediation of dyes effluent. Nevertheless, it is too slow in achieving the desired removal state. Other removal strategies such as membrane separation and oxidation processes demonstrate barriers in operating and 
maintenance costs, efficiency, and secondary waste generation which also prompts additional handling and disposal requirements.

Adsorption has been widely recognized as the effective method to abate dye wastewater even at low concentration. In addition, the process is simple, inexpensive and easy to scale-up. Now, it continues in attracting research for the improvement of dyes-laden wastewater treatment. Recent studies show several attempts to boost the adsorption performance of malachite green and congo red dyes by various adsorbents with special characters while performing the process at the optimum operating conditions. In this short review, recent literature on the theme in 10 years back is reviewed and summarized to bring new insight for future research. All published studies cited in this paper are based on laboratory scale batch adsorption which is also known as bottle-point-technique using simulated single component dye solutions.

\section{Recent studies on the adsorption of malachite green and congo red from water}

Tables 2 and 3 summarize the adsorption of malachite green and congo red dyes from water by various adsorbents. In general, direct relationships between the maximum adsorption capacity of dyes and the characteristics of adsorbents could be established. Particularly, the surface area, mesoporosity and solution $\mathrm{pH}$ exhibit a positive effect on the adsorption performance. A higher surface area generally provides a greater number of available active sites and improved interaction probabilities for adsorption. Moreover, the mesoporous adsorbents with pore size ranging between 2 and $50 \mathrm{~nm}$ are more preferred because dye molecules could easily penetrate and lodge onto the channel textures. In addition, the surface chemistry and solution $\mathrm{pH}$ could play a considerable role in adsorption depending on the charge density of dyes. For example, the cationic dye adsorption would favour the process at high solution $\mathrm{pH}$ using adsorbent rich in surface acidic oxygen groups for dual electrostatic attraction mechanisms, i.e., deprotonated adsorbent surface and dissociated functional groups.

Table 2 shows the adsorbents with special characters that have been employed to remove malachite green from simulated waste water with adsorption capacity ranging from 20 to $1265 \mathrm{mg} / \mathrm{g}$. The oxidized mesoporous carbon with a surface area of $334 \mathrm{~m}^{2} / \mathrm{g}$ (61\% mesoporosity) displays a higher malachite green adsorption capacity of $1265 \mathrm{mg} / \mathrm{g}$ [8]. The mesoporous carbon has attracted an increasing attention for malachite green adsorption, due to its sufficient pore entrance to accommodate dye molecules, large specific surface area and pore volume, excellent surface chemistry and thermal stability. The adsorption capacity is higher even though the surface area is not as superior as the other counterparts. Therefore, the mechanisms of removal could be attributed not only to pore filling, but also surface complexation with oxygen groups and $\pi$ - $\pi$ interactions as visualized in Figures 1 and 2.

In general, the building matrix, functional groups and mesoporous nature of adsorbent offer the synergistic interactions with cationic dyes that surely assist in enhancing their removal from wastewater. The strong polarizing effect of nitrogen atom of malachite green molecule renders an electron-deficient $\pi$ system in its aromatic ring. Consequently, the tertiary amine group gains a high dipole moment for electrostatic interaction with the negatively charged, dissociated oxygen functional groups in the adsorbent surface. 
Meanwhile, the cationic centre of dye molecule also develops a non-covalent dipole- $\pi$ interaction with delocalized $\pi$-electron clouds of graphitic-based adsorbent.

Table 2

Adsorption of malachite green dye by various adsorbents

\begin{tabular}{|c|c|c|c|c|c|c|c|}
\hline Adsorbents & $\begin{array}{c}\text { Surface } \\
\text { area } \\
{\left[\mathrm{m}^{2} / \mathrm{g}\right]} \\
\end{array}$ & \begin{tabular}{|c|} 
Mesopo- \\
rosity \\
{$[\%]$} \\
\end{tabular} & $\begin{array}{l}\text { Pore } \\
\text { width } \\
\text { [nm] }\end{array}$ & $\begin{array}{c}\text { Maximum } \\
\text { adsorption } \\
\text { capacity [mg/g] }\end{array}$ & \begin{tabular}{|c|c}
$\begin{array}{c}\text { Affinity of } \\
\text { adsorption } \\
{\left[\mathrm{dm}^{3} / \mathrm{mg}\right]}\end{array}$ \\
\end{tabular} & $\begin{array}{l}\mathbf{p H} \\
{[-]}\end{array}$ & $\begin{array}{l}\text { Refe- } \\
\text { rence }\end{array}$ \\
\hline Hemicellulose-based adsorbent & - & - & - & 96.1 & - & 6.5 & {$[1]$} \\
\hline $\begin{array}{l}\text { Magnetic-cyclodextrin-graphene oxide } \\
\text { nanocomposites }\end{array}$ & - & - & - & 741 & 0.0115 & 7 & {$[3]$} \\
\hline Oxidized mesoporous carbon & 334 & 61 & 3.9 & 1265 & 0.051 & 6.5 & {$[8]$} \\
\hline Reduced graphene oxide & 931 & - & 3.0 & 476 & 1.75 & 6 & [9] \\
\hline $\begin{array}{l}\text { Carboxylate group-functionalized } \\
\text { multi-walled carbon nanotubes }\end{array}$ & 400 & - & 1.5 & 11.8 & 0.120 & 9 & {$[13,14]$} \\
\hline $\begin{array}{c}\text { Potassium salts-activated } \\
\text { carbons from textile sludge }\end{array}$ & 481 & 57 & 34 & 167 & 1.67 & 6 & [15] \\
\hline Magnetic $\mathrm{CuFe}_{2} \mathrm{O}_{4}$ nano-adsorbent & 128 & - & 1.8 & 197 & 0.220 & 5.4 & {$[16]$} \\
\hline $\begin{array}{c}\text { Starch-graft- } \\
\text { poly(acrylamide)/hydrogels } \\
\end{array}$ & - & - & - & 287 & 0.642 & 5.5 & [17] \\
\hline Sulfonic acid-modified coal fly ash & 69.4 & - & 3.0 & 233 & 0.481 & - & {$[18]$} \\
\hline $\begin{array}{l}\text { Zeolite nanostructures from waste } \\
\text { aluminium cans }\end{array}$ & - & - & - & 227 & 0.112 & - & [19] \\
\hline Bio-based magnetic activated carbon & 389 & 69 & 4.6 & 218 & 0.061 & 6 & {$[20]$} \\
\hline Fibrous cellulose sulfate & 36.6 & - & & 960 & 0.240 & 6 & [21] \\
\hline $\begin{array}{l}\text { Tetraethylenepentamine-functionalized } \\
\text { activated carbon }\end{array}$ & - & - & & 333 & 0.319 & 8 & {$[22]$} \\
\hline $\begin{array}{l}\text { Lignin sulfonate-based mesoporous } \\
\text { materials }\end{array}$ & 118 & - & 3.8 & 121 & 0.269 & 7 & [23] \\
\hline $\begin{array}{l}\text { Nickel hydroxide nanoplates-modified } \\
\text { activated carbon }\end{array}$ & 960 & 87 & 3.5 & 76.9 & 1.34 & 6.5 & [24] \\
\hline $\begin{array}{c}\text { ZnO nanorod-loaded } \\
\text { activated carbon } \\
\end{array}$ & - & - & & 20 & 10.6 & 6 & [25] \\
\hline $\begin{array}{c}\text { Copper nanowires } \\
\text { loaded on activated carbon }\end{array}$ & 689 & - & 7.4 & 164 & 0.239 & 5 & [26] \\
\hline $\begin{array}{c}\text { Steam } / \mathrm{H}_{3} \mathrm{PO}_{4} \text {-activated carbon } \\
\text { produced from waste printed circuit } \\
\text { boards }\end{array}$ & 730 & 40.2 & 2.5 & 769 & 1.18 & - & [27] \\
\hline $\begin{array}{l}\text { Magnetic metal organic framework } \\
\text { composite }\end{array}$ & 35.4 & - & - & 114 & - & - & [28] \\
\hline
\end{tabular}

Table 3

Adsorption of congo red dye by various adsorbents

\begin{tabular}{|c|c|c|c|c|c|c|c|}
\hline Adsorbent & $\begin{array}{c}\text { Surface } \\
\text { area } \\
{\left[\mathbf{m}^{2} / \mathbf{g}\right]}\end{array}$ & $\begin{array}{c}\text { Mesopo- } \\
\text { rosity [\%] }\end{array}$ & $\begin{array}{c}\text { Pore } \\
\text { width } \\
{[\mathbf{n m}]}\end{array}$ & $\begin{array}{c}\text { Maximum } \\
\text { adsorption } \\
\text { capacity }[\mathbf{m g} / \mathbf{g}]\end{array}$ & $\begin{array}{c}\text { Affinity of } \\
\mathbf{a d s o r p t i o n} \\
{[\mathbf{d m} \mathbf{3} / \mathbf{m g}]}\end{array}$ & $\begin{array}{c}\mathbf{p H} \\
{[-]}\end{array}$ & $\begin{array}{c}\text { Refe- } \\
\text { rence }\end{array}$ \\
\hline Zeolitic imidazolate & 1388 & - & & 714 & 0.272 & 7 & {$[6]$} \\
\hline Resorcinol formaldehyde carbon gel & 639 & 69.8 & 6.9 & 2.25 & 25.6 & 5.4 & {$[10]$} \\
\hline $\begin{array}{c}\mathrm{HNO}_{3} \text {-modified resorcinol } \\
\text { formaldehyde carbon gel }\end{array}$ & 711 & 75.1 & 7.7 & 10.6 & 0.720 & 5.4 & {$[10]$} \\
\hline Shrimp shell powder & 66.4 & 92.1 & 5.6 & 232 & 0.0463 & 5.5 & {$[29]$} \\
\hline Vermicompost - derived biochars & 76.3 & - & 9.9 & 31.3 & 0.0832 & 7 & {$[30]$} \\
\hline Zinc peroxide nanomaterial & - & - & - & 208 & - & $2-$ & {$[31]$} \\
\hline
\end{tabular}




\begin{tabular}{|c|c|c|c|c|c|c|c|}
\hline Adsorbent & $\begin{array}{c}\text { Surface } \\
\text { area } \\
{\left[\mathbf{m}^{2} / \mathbf{g}\right]}\end{array}$ & $\begin{array}{l}\text { Mesopo- } \\
\text { rosity [\%] }\end{array}$ & $\begin{array}{l}\text { Pore } \\
\text { width } \\
{[\mathrm{nm}]}\end{array}$ & \begin{tabular}{|c|} 
Maximum \\
adsorption \\
capacity $[\mathrm{mg} / \mathrm{g}]$
\end{tabular} & $\begin{array}{c}\text { Affinity of } \\
\text { adsorption } \\
{\left[\mathrm{dm}^{3} / \mathrm{mg}\right]}\end{array}$ & $\begin{array}{l}\text { pH } \\
{[-]}\end{array}$ & $\begin{array}{l}\text { Refe- } \\
\text { rence }\end{array}$ \\
\hline $\begin{array}{l}\text { Superparamagnetic CTAB-modified } \\
\text { magnetite nanoparticles }\end{array}$ & 109 & - & & 93.5 & 0.117 & - & {$[32]$} \\
\hline $\mathrm{MgBO}_{2}(\mathrm{OH})$ microspheres & 94.8 & - & 34.2 & 309 & 3.85 & - & [33] \\
\hline $\begin{array}{c}\text { Ionic liquid-based nanoporous } \\
\text { organosilica } \\
\text { supported propylamine }\end{array}$ & 586 & - & 9.9 & 43.1 & 1.28 & 4 & {$[34]$} \\
\hline $\begin{array}{c}\text { Flower-like } \\
\text { hierarchical magnesium oxide- } \\
\text { graphene oxide composite } \\
\text { microspheres }\end{array}$ & 109 & - & 17.4 & 237 & 1.46 & 6.5 & [35] \\
\hline Post-consumer waste polystyrene & 752 & - & 8.69 & 500 & 0.00197 & 6.9 & [36] \\
\hline Natural serpentine & 43.6 & - & - & 93.5 & 0.00182 & - & [37] \\
\hline $\begin{array}{c}\text { Activated carbon } \\
\text { with electropositive amine } \\
\text { modification }\end{array}$ & 22.8 & - & - & 319 & 1.44 & 7 & [38] \\
\hline Ag-doped hydroxyapatite & 101 & - & - & 555 & 0.0022 & - & [39] \\
\hline $\begin{array}{c}\text { Sonochemically synthesized } \mathrm{NiAl} \\
\text { layered double hydroxide }\end{array}$ & 29 & - & 3.2 & 121 & 0.346 & 6 & {$[40]$} \\
\hline Porous hierarchical $\alpha-\mathrm{Ni}(\mathrm{OH})_{2}$ & 191 & - & 19.5 & 206 & 49.7 & - & [41] \\
\hline MWCNTs/LDHs nanohybrids & 125 & - & 3.8 & 596 & - & 5 & {$[42]$} \\
\hline $\begin{array}{l}\text { Monodispersed hierarchical NiO } \\
\text { microspheres }\end{array}$ & 107 & - & 7.0 & 535 & 0.840 & - & {$[43]$} \\
\hline $\begin{array}{c}\mathrm{Fe}_{3} \mathrm{O}_{4} @ \mathrm{nSiO}_{2} @ \mathrm{mSiO}_{2} \text { core-shell } \\
\text { microspheres }\end{array}$ & 38.2 & - & 15.3 & 1429 & 0.259 & 5.5 & {$[44]$} \\
\hline Flower-like $\mathrm{ZnO}-\mathrm{AlOOH}$ & 211 & - & 9.5 & 524 & 1.09 & 6.5 & [45] \\
\hline $\begin{array}{l}\text { Quercetin modified } \alpha-\mathrm{Fe}_{2} \mathrm{O}_{3} \\
\text { nanoparticles }\end{array}$ & 86.9 & - & 98.6 & 427 & 1.72 & 5.4 & [46] \\
\hline
\end{tabular}

a)

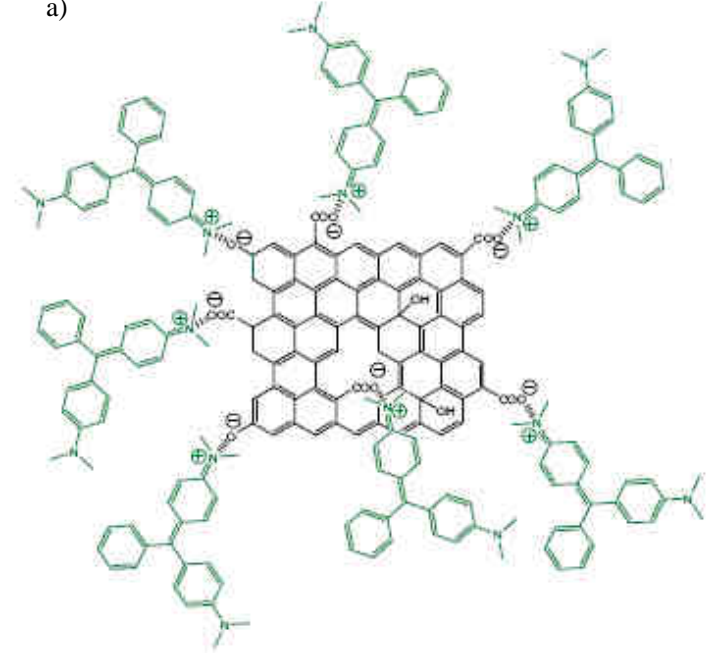

b)

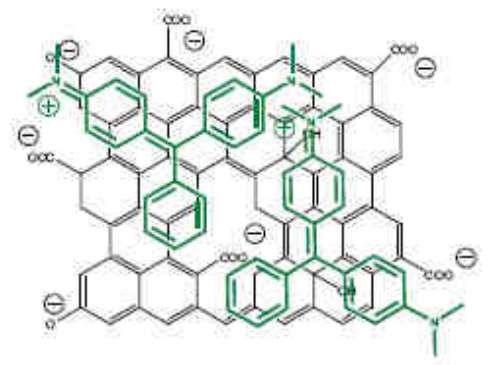

Fig. 1. Adsorption mechanisms of malachite green by graphitic-based adsorbent rich with carboxylic functional groups: a) electrostatic interaction between the cationic centre of dye molecule and negatively charged carboxylic groups of adsorbent, and b) $\pi-\pi$ interaction driven by the $\pi$-electrons cloud of adsorbent and aromatic rings of dye molecule (Adapted from [9] with permission) 


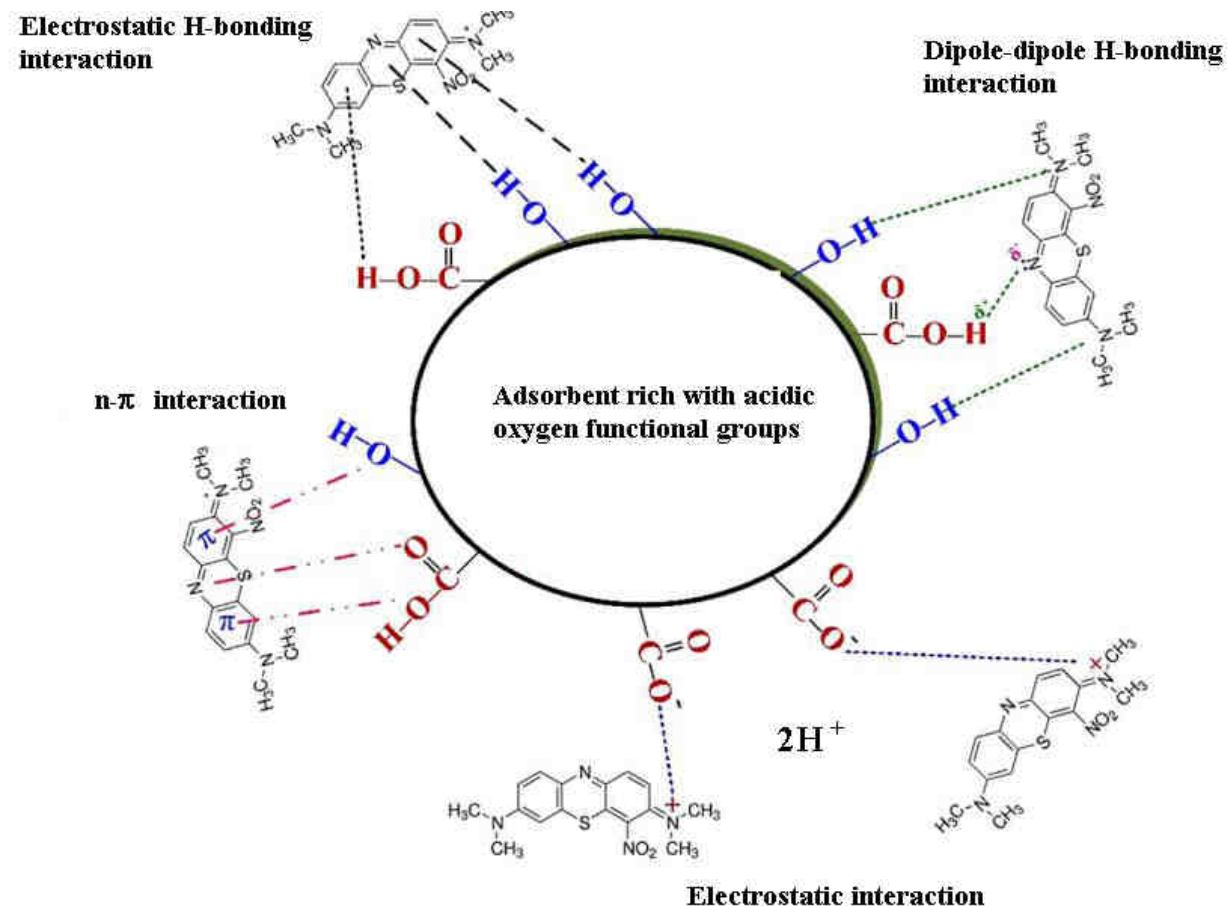

Fig. 2. Interactions between cationic dye molecules with adsorbent rich with acidic oxygen functional groups (adapted from [47] with permission)

The adsorption of malachite green normally obeyed Langmuir model, suggesting a monolayer type of adsorption on homogeneous adsorbent surface. Some malachite green adsorbents exhibit high adsorption affinity in the range of 1.18 to $10.6 \mathrm{dm}^{3} / \mathrm{mg}$ $[9,15,24,25,27]$. However, the magnitude does not necessarily means a superior dyes removal. The affinity for malachite green adsorption by oxidized mesoporous carbon is relatively small at $0.051 \mathrm{dm}^{3} / \mathrm{mg}$ as compared with other adsorbents [8]. It implies that the adsorption of malachite green is mainly driven by concentration, and may not be suitable for adsorption at low concentration. It should be noted, however, the application of oxidized mesoporous carbon for wastewater treatment is limited by its high cost and small-scale production [8]. In a related development, Gupta and Khatri [9] reported a removal of $476 \mathrm{mg} / \mathrm{g}$ malachite green by graphene oxide with a surface area of $931 \mathrm{~m}^{2} / \mathrm{g}$. The three-dimensional graphene microstructure allows the accumulation of dye molecules, hence increasing the adsorption capacity [9]. In a related work, Kan and co-workers [27] reported a $769 \mathrm{mg} / \mathrm{g}$ malachite green removal by steam $/ \mathrm{H}_{3} \mathrm{PO}_{4}$-activated carbon produced from waste printed circuit boards $730 \mathrm{~m}^{2} / \mathrm{g}$ (40.2\% mesoporosity). On the other hand, a high surface area nickel hydroxide nanoplates-modified activated carbon $\left(960 \mathrm{~m}^{2} / \mathrm{g}\right.$, $87 \%$ mesoporosity) reveals only a $76.9 \mathrm{mg} / \mathrm{g}$ of malachite green removal [24]. It signifies that the textural characteristics alone may not be enough to boost the performance of adsorbents for dyes removal. For example, carboxylate group-functionalized multi-walled carbon nanotubes $\left(400 \mathrm{~m}^{2} / \mathrm{g}\right.$, pore width $\left.1.5 \mathrm{~nm}\right)$ shows only a $11.8 \mathrm{mg} / \mathrm{g}$ of malachite green removal at $\mathrm{pH}=9$ due to its microporous texture and narrow pore width to allow the 
transport of dye molecules even the adsorbent is pre-functionalized [13]. Similarly, a microporous magnetic $\mathrm{CuFe}_{2} \mathrm{O}_{4}$ nano-adsorbent $\left(128 \mathrm{~m}^{2} / \mathrm{g}\right.$, pore width $\left.1.8 \mathrm{~nm}\right)$ exhibits an adsorption of $197 \mathrm{mg} / \mathrm{g}$ at $\mathrm{pH}=5.4$ [16]. On a side note, Tang and Zaini [10] reported that the $\mathrm{HNO}_{3}$-modified resorcinol formaldehyde carbon gel with a surface area of $711 \mathrm{~m}^{2} / \mathrm{g}$ displays a higher adsorption capacity compared to the non-modified one. The burnout of organic matters during carbonization gives rise to the formation of new voids, thus increasing the surface area, mesoporosity and average pore diameter of carbon gel for dye adsorption. In addition, post-oxidation and post-functionalization processes of mesoporous adsorbents are expected to introduce surface acidic oxygen groups that are beneficial to enhance the removal of cationic dyes [8]. Figure 3 illustrates the possible interactions between malachite green molecules with adsorbent functionalized with sulfonic acid [18].

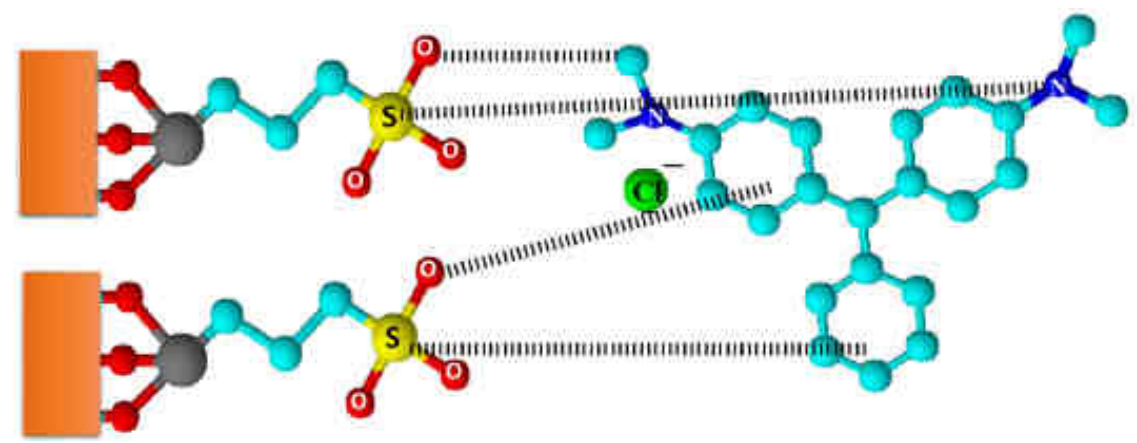

Fig. 3. Adsorption mechanisms of malachite green by sulfonic acid functionalized adsorbent (adapted from [18] with permission)

Table 3 summarizes the recently used congo red adsorbents with adsorption capacity in the range of 2.25 to $1429 \mathrm{mg} / \mathrm{g}$. Some adsorbents with surface area between 100 and $200 \mathrm{~m}^{2} / \mathrm{g}$ demonstrate the congo red removal of $500 \mathrm{mg} / \mathrm{g}$ to $600 \mathrm{mg} / \mathrm{g}$ [39, 42, 43, 45]. The increase in mesoporosity improves the adsorption of congo red [10]. Among the studied adsorbents, the porous hierarchical $\alpha-\mathrm{Ni}(\mathrm{OH})_{2}$ with surface area of $191 \mathrm{~m}^{2} / \mathrm{g}$ yields a higher affinity of $49.7 \mathrm{dm}^{3} / \mathrm{mg}$, even though the removal capacity is small at $206 \mathrm{mg} / \mathrm{g}$ [41]. From Table 3, Tu et al. [6] reported the removal of congo red onto zeolitic imidazolate framework derived from tetrahedral metal ions of $\mathrm{Zn}$ and Co. The adsorbent exhibits a surface area of $1388 \mathrm{~m}^{2} / \mathrm{g}$ for the adsorption capacity of $714 \mathrm{mg} / \mathrm{g}$ at $\mathrm{pH}=7$ [6]. Despite the fact that a large surface area normally renders a high adsorption capacity, Zhang and co-workers [44] revealed a superior removal of $1429 \mathrm{mg} / \mathrm{g}$ congo red by $\mathrm{Fe}_{3} \mathrm{O}_{4} @ \mathrm{nSiO}_{2} @ \mathrm{mSiO}_{2}$ core-shell microspheres with a small surface area of $38.2 \mathrm{~m}^{2} / \mathrm{g}$. The adsorption mechanism could be associated to electrostatic attraction between the positively charged surface and negatively charged dye molecules, and $\pi-\pi$ interactions. The adsorbent material shows a promising potential in anionic dye adsorption because of a relatively high adsorption capacity and an easy separation using external magnetic field.

Leyva-Ramos [11] reported that an extreme increase or decrease in solution $\mathrm{pH}$ to a certain extent would result in the decrease of adsorption capacity due to the suppression of counter ions and competition for active sites, irrespective of the charge density of dyes. Hence, the adsorption is normally carried out at a moderate solution $\mathrm{pH}$ to yield a high 
adsorption capacity. From Tables 2 and 3, magnetic-cyclodextrin-graphene oxide nanocomposites [3], reduced graphene oxide [9], tetraethylenepentamine functionalized activated carbon [22], copper nanowires loaded on activated carbon [26], and post-consumer waste polystyrene [36] demonstrate a high adsorption capacity at slightly low or neutral $\mathrm{pH}$. Nevertheless, there are exceptional cases whereby the adsorption is $\mathrm{pH}$-independent and unaffected with the change of solution $\mathrm{pH}$. According to Nouri [12], the affinity for adsorption is directly proportional to the $\mathrm{pH}$ of adsorbent. When the $\mathrm{pH}$ of adsorbent is high, the affinity of dyes adsorption would also increase, depending on how the solution $\mathrm{pH}$ is manipulated to suit the charge density of dyes.

Table 4 summarizes the operating parameters for malachite green and congo red dyes adsorption by various adsorbents. The dosage of adsorbent is ranging between 0.1 and $3.33 \mathrm{~g} / \mathrm{dm}^{3}$. In general, a maximum of $1 \mathrm{~g} / \mathrm{dm}^{3}$ dosage is applied in batch adsorption in order to minimize the increase of solution volume that could compromise the material balance for which the volume is assumed constant throughout the process. The equilibrium adsorption for an adsorbent at certain concentration depends on adsorbent dosage. A greater dosage endows a higher adsorption capacity to a maximum or saturation point, before it deteriorates due to exhaustive adsorption along with an increased magnitude of adsorbent dosage for a certain concentration of dyes.

Table 4

Rate constant and thermodynamics of dyes adsorption by various adsorbents

\begin{tabular}{|c|c|c|c|c|c|c|c|}
\hline Adsorbents & $\begin{array}{l}\text { Dosage } \\
{\left[\mathrm{g} / \mathrm{dm}^{3}\right]}\end{array}$ & \begin{tabular}{|c|} 
Dye \\
concen- \\
tration \\
{$\left[\mathrm{mg} / \mathbf{d m}^{3}\right]$}
\end{tabular} & $\begin{array}{c}\text { Equili- } \\
\text { brium } \\
\text { time } \\
\text { [min] }\end{array}$ & $\begin{array}{c}\text { Equili- } \\
\text { brium } \\
\text { adsorption } \\
{[\mathrm{mg} / \mathrm{g}]}\end{array}$ & $\begin{array}{c}\text { Rate } \\
\text { constant } \\
[\text { g/(mg'min })]\end{array}$ & $\begin{array}{l}\text { Thermo- } \\
\text { dynamics }\end{array}$ & $\begin{array}{l}\text { Refe- } \\
\text { rence }\end{array}$ \\
\hline \multicolumn{8}{|c|}{ Malachite green } \\
\hline Hemicellulose-based adsorbent & 1 & 100 & 90 & 96.1 & $1.68 \cdot 10^{-3}$ & $\begin{array}{l}\text { Endo- } \\
\text { thermic }\end{array}$ & [1] \\
\hline $\begin{array}{l}\text { Magnetic-cyclodextrin-graphene } \\
\text { oxide nanocomposites }\end{array}$ & 0.25 & 10 & 100 & 35.0 & $3.70 \cdot 10^{-3}$ & $\begin{array}{l}\text { Endo- } \\
\text { thermic }\end{array}$ & [3] \\
\hline Oxidized mesoporous carbon & 0.5 & 500 & $1.2 \cdot 10^{3}$ & 943 & 71.6 & $\begin{array}{l}\text { Exo- } \\
\text { thermic }\end{array}$ & {$[8]$} \\
\hline \multirow{2}{*}{ Reduced graphene oxide } & \multirow{2}{*}{0.5} & 100 & 200 & 186 & $2.60 \cdot 10^{-4}$ & \multirow{2}{*}{$\begin{array}{l}\text { Endo- } \\
\text { thermic }\end{array}$} & \multirow{2}{*}{ [9] } \\
\hline & & 200 & 600 & 412 & $3.70 \cdot 10^{-5}$ & & \\
\hline $\begin{array}{l}\text { Carboxylate group-functionalized } \\
\text { multi-walled carbon nanotubes }\end{array}$ & 1 & 20 & 10 & 11.8 & 0.181 & $\begin{array}{l}\text { Endo- } \\
\text { thermic }\end{array}$ & [13] \\
\hline \multirow{2}{*}{$\begin{array}{l}\text { Potassium salts-activated } \\
\text { carbons from textile sludge }\end{array}$} & \multirow{2}{*}{1} & 150 & $3.60 \cdot 10^{3}$ & 137 & $5.83 \cdot 10^{-5}$ & \multirow{2}{*}{$\begin{array}{l}\text { Endo- } \\
\text { thermic }\end{array}$} & \multirow{2}{*}[15]{} \\
\hline & & 400 & $4.20 \cdot 10^{3}$ & 156 & $3.83 \cdot 10^{-5}$ & & \\
\hline Magnetic $\mathrm{CuFe}_{2} \mathrm{O}_{4}$ nano-adsorbent & 0.3 & 10 & 50 & 26.7 & - & - & {$[16]$} \\
\hline $\begin{array}{c}\text { Starch-graft- } \\
\text { poly(acrylamide)/hydrogels }\end{array}$ & 1 & 150 & - & 276 & $6.70 \cdot 10^{-3}$ & $\begin{array}{l}\text { Endo- } \\
\text { thermic }\end{array}$ & [17] \\
\hline \multirow{2}{*}{$\begin{array}{l}\text { Sulfonic acid-modified coal fly } \\
\text { ash }\end{array}$} & \multirow{2}{*}{1.6} & 100 & 40 & 169 & $3.80 \cdot 10^{-3}$ & \multirow{2}{*}{$\begin{array}{l}\text { Endo- } \\
\text { thermic }\end{array}$} & \multirow{2}{*}{ [18] } \\
\hline & & 150 & 40 & 249 & $2.80 \cdot 10^{-3}$ & & \\
\hline $\begin{array}{l}\text { Zeolite nanostructures from waste } \\
\text { aluminium cans }\end{array}$ & 1 & 700 & 200 & 227 & $6.30 \cdot 10^{-5}$ & $\begin{array}{c}\text { Exo- } \\
\text { thermic }\end{array}$ & [19] \\
\hline $\begin{array}{c}\text { Bio-based magnetic activated } \\
\text { carbon }\end{array}$ & 1 & 100 & 60 & 92.5 & - & $\begin{array}{l}\text { Endo- } \\
\text { thermic }\end{array}$ & [20] \\
\hline \multirow{2}{*}{ Fibrous cellulose sulphate } & \multirow{2}{*}{0.2} & 100 & 10 & 481 & $5.30 \cdot 10^{-3}$ & \multirow{2}{*}{$\begin{array}{c}\text { Exo- } \\
\text { thermic }\end{array}$} & \multirow{2}{*}{ [21] } \\
\hline & & 200 & 20 & 930 & $1.50 \cdot 10^{-3}$ & & \\
\hline $\begin{array}{c}\text { Tetraethylenepentamine- } \\
\text { functionalized activated carbon }\end{array}$ & 0.2 & 50 & 60 & 196 & $7.00 \cdot 10^{-3}$ & $\begin{array}{l}\text { Endo- } \\
\text { thermic }\end{array}$ & [22] \\
\hline
\end{tabular}




\begin{tabular}{|c|c|c|c|c|c|c|c|}
\hline Adsorbents & $\begin{array}{l}\text { Dosage } \\
{\left[\mathrm{g} / \mathrm{dm}^{3}\right]}\end{array}$ & $\begin{array}{c}\text { Dye } \\
\text { concen- } \\
\text { tration } \\
{\left[\mathrm{mg} / \mathbf{d m}^{3}\right]}\end{array}$ & $\begin{array}{l}\text { Equili- } \\
\text { brium } \\
\text { time } \\
\text { [min] }\end{array}$ & \begin{tabular}{|c|} 
Equili- \\
brium \\
adsorption \\
{$[\mathrm{mg} / \mathrm{g}]$}
\end{tabular} & $\begin{array}{c}\text { Rate } \\
\text { constant } \\
[\text { g/(mg'min })]\end{array}$ & $\begin{array}{l}\text { Thermo- } \\
\text { dynamics }\end{array}$ & $\begin{array}{l}\text { Refe- } \\
\text { rence }\end{array}$ \\
\hline \multirow{2}{*}{$\begin{array}{c}\text { Lignin sulfonate-based } \\
\text { mesoporous materials }\end{array}$} & \multirow{2}{*}{1} & 40 & 115 & 37.8 & $6.11 \cdot 10^{-4}$ & \multirow{2}{*}{$\begin{array}{l}\text { Endo- } \\
\text { thermic }\end{array}$} & \multirow{2}{*}[23]{} \\
\hline & & 100 & 200 & 98.5 & $1.60 \cdot 10^{-3}$ & & \\
\hline \multirow{2}{*}{$\begin{array}{l}\text { Nickel hydroxide nanoplates- } \\
\text { modified activated carbon }\end{array}$} & \multirow{2}{*}{0.7} & 10 & 13 & 14.0 & 0.320 & \multirow{2}{*}{$\begin{array}{l}\text { Endo- } \\
\text { thermic }\end{array}$} & \multirow{2}{*}{ [24] } \\
\hline & & 20 & 20 & 29.1 & 0.047 & & \\
\hline \multirow{2}{*}{$\begin{array}{l}\text { ZnO nanorod-loaded } \\
\text { activated carbon }\end{array}$} & \multirow{2}{*}{0.6} & 8 & $<4$ & 14.1 & 0.0578 & \multirow[b]{2}{*}{-} & \multirow{2}{*}[25]{} \\
\hline & & 12 & $<4$ & 22.0 & 0.0352 & & \\
\hline \multicolumn{8}{|c|}{ Congo red } \\
\hline \multirow{2}{*}{ Zeolitic imidazolate } & \multirow{2}{*}{0.2} & 50 & 25 & 445 & 0.017 & \multirow{2}{*}{$\begin{array}{l}\text { Endo- } \\
\text { thermic }\end{array}$} & \multirow{2}{*}{ [6] } \\
\hline & & 80 & 40 & 628 & 0.013 & & \\
\hline \multirow{2}{*}{$\begin{array}{l}\text { Resorcinol formaldehyde carbon } \\
\text { gel }\end{array}$} & \multirow{2}{*}{1.5} & 5 & - & 2.94 & $2.55 \cdot 10^{-4}$ & \multirow{2}{*}{ - } & \multirow{2}{*}[10]{} \\
\hline & & 10 & \begin{tabular}{|l|}
- \\
\end{tabular} & 3.58 & $1.70 \cdot 10^{-4}$ & & \\
\hline \multirow{2}{*}{$\begin{array}{l}\mathrm{HNO}_{3} \text {-modified resorcinol } \\
\text { formaldehyde carbon gel }\end{array}$} & \multirow{2}{*}{1.5} & 5 & $3.00 \cdot 10^{3}$ & 3.19 & $3.00 \cdot 10^{-3}$ & \multirow{2}{*}{-} & \multirow{2}{*}[10]{} \\
\hline & & 10 & $4.20 \cdot 10^{3}$ & 4.73 & $6.33 \cdot 10^{-3}$ & & \\
\hline $\begin{array}{c}\text { Shrimp } \\
\text { shell powder }\end{array}$ & 3.33 & 500 & 700 & 144 & $2.77 \cdot 10^{-4}$ & $\begin{array}{l}\text { Endo- } \\
\text { thermic }\end{array}$ & [29] \\
\hline $\begin{array}{l}\text { Vermicompost- } \\
\text { derived biochars }\end{array}$ & 1.5 & 25 & 75 & 14.3 & 0.021 & - & [30] \\
\hline $\mathrm{MgBO}_{2}(\mathrm{OH})$ microspheres & 0.5 & 100 & 150 & 200 & $2.40 \cdot 10^{-3}$ & - & [33] \\
\hline $\begin{array}{c}\text { Ionic liquid-based nanoporous } \\
\text { organosilica } \\
\text { supported propylamine }\end{array}$ & 0.5 & 40 & 33 & 22.7 & $8.00 \cdot 10^{-3}$ & $\begin{array}{l}\text { Endo- } \\
\text { thermic }\end{array}$ & {$[34]$} \\
\hline $\begin{array}{l}\text { Flower-like hierarchical } \\
\text { magnesium oxide-graphene oxide } \\
\text { composite microspheres }\end{array}$ & 0.2 & 50 & 55 & 230 & $4.30 \cdot 10^{-3}$ & - & {$[35]$} \\
\hline Natural serpentine & 2 & 100 & 200 & 15.4 & $2.50 \cdot 10^{-3}$ & $\begin{array}{l}\text { Exo- } \\
\text { thermic }\end{array}$ & {$[37]$} \\
\hline $\begin{array}{c}\text { Activated carbon with } \\
\text { electropositive amine modification }\end{array}$ & 0.5 & 100 & 50 & 194 & $2.70 \cdot 10^{-5}$ & - & {$[38]$} \\
\hline \multirow{2}{*}{ Ag-doped hydroxyapatite } & \multirow{2}{*}{1} & 50 & 20 & 49.8 & 0.533 & \multirow[b]{2}{*}{ - } & \multirow{2}{*}{ [39] } \\
\hline & & 300 & 600 & 280 & $8.33 \cdot 10^{-4}$ & & \\
\hline \multirow{2}{*}{$\begin{array}{c}\text { Sonochemically Synthesized NiAl } \\
\text { layered double hydroxide }\end{array}$} & 05 & 10 & 30 & 31.9 & 0.016 & Endo- & {$[40]$} \\
\hline & 0.5 & 30 & 169 & 79.9 & $4.50 \cdot 10^{-4}$ & thermic & {$[40]$} \\
\hline MWCNTs/LDHs nanohybrids & 0.13 & 150 & 300 & 599 & 1.00 & $\begin{array}{l}\text { Endo- } \\
\text { thermic }\end{array}$ & {$[42]$} \\
\hline $\begin{array}{l}\text { Monodispersed hierarchical NiO } \\
\text { microspheres }\end{array}$ & 0.1 & 50 & 120 & 455 & $5.70 \cdot 10^{-4}$ & $\begin{array}{l}\text { Endo- } \\
\text { thermic }\end{array}$ & [43] \\
\hline $\mathrm{Fe}_{3} \mathrm{O}_{4} @ \mathrm{nSiO}_{2} @ \mathrm{mSiO}_{2}$ core-shell & 033 & 400 & 50 & 1111 & $1.76 \cdot 10^{-4}$ & Exo- & {$[44]$} \\
\hline microspheres & 0.33 & 600 & 100 & 1429 & $7.60 \cdot 10^{-5}$ & thermic & [44] \\
\hline Flower-like $\mathrm{ZnO}-\mathrm{AlOOH}$ & 0.1 & 50 & 250 & 429 & $1.71 \cdot 10^{-4}$ & - & {$[45]$} \\
\hline $\begin{array}{l}\text { Quercetin modified } \alpha-\mathrm{Fe}_{2} \mathrm{O}_{3} \\
\text { Nanoparticles }\end{array}$ & 1.4 & 35 & 140 & 541 & $1.30 \cdot 10^{-4}$ & $\begin{array}{l}\text { Endo- } \\
\text { thermic }\end{array}$ & [46] \\
\hline
\end{tabular}

From Table 4, the time taken to reach equilibrium is ranging from few minutes to more than $24 \mathrm{~h}$. A fast adsorption to reach equilibrium relies on dosage and concentration. The mutual interaction depends on concentration gradient as driving force, either higher concentration with limited active sites, or lower concentration with higher active sites. Nevertheless, the external factor of adsorbent inherent properties could also promote rapid adsorption. The oxidized mesoporous carbon takes nearly $20 \mathrm{~h}$ to attain equilibrium for malachite green concentration of $500 \mathrm{mg} / \mathrm{dm}^{3}$ with equilibrium capacity of $943 \mathrm{mg} / \mathrm{g}$ [8]. By using the same dosage of $0.5 \mathrm{~g} / \mathrm{dm}^{3}$, the reduced graphene oxide takes about $10 \mathrm{~h}$ to 
achieve equilibrium at capacity of $412 \mathrm{mg} / \mathrm{g}$ for dye concentration of $200 \mathrm{mg} / \mathrm{dm}^{3}$ [9]. Meanwhile, fibrous cellulose sulphate [21] exhibits a more rapid equilibrium of $20 \mathrm{~min}$ at $930 \mathrm{mg} / \mathrm{g}$ using a smaller dosage of $0.2 \mathrm{~g} / \mathrm{dm}^{3}$ and dye concentration of $200 \mathrm{mg} / \mathrm{dm}^{3}$. Similarly, $\mathrm{Fe}_{3} \mathrm{O}_{4} @ \mathrm{nSiO}_{2} @ \mathrm{mSiO}_{2}$ core-shell microspheres [44] demonstrates a superior adsorption of $1429 \mathrm{mg} / \mathrm{g}$ at a rapid pace of $100 \mathrm{~min}$ for congo red concentration of $600 \mathrm{mg} / \mathrm{dm}^{3}$ and $0.33 \mathrm{~g} / \mathrm{dm}^{3}$ dosage. On the other hand, Tang and Zaini [15] reported a longer contact time to reach equilibrium at $60 \mathrm{~h}$ and $70 \mathrm{~h}$ for congo red concentrations of 150 and $400 \mathrm{mg} / \mathrm{dm}^{3}$, respectively. A longer contact time for an increase in dyes concentration has been reported in a number of studies [6, 9, 10, 21, 23, 24, 39, 40, 44]. For example, the equilibrium time increases from $20 \mathrm{~min}$ to $10 \mathrm{~h}$ with increasing congo red concentration from 50 to $300 \mathrm{mg} / \mathrm{dm}^{3}$ by $\mathrm{Ag}$-doped hydroxyapatite adsorption [39]. Likewise, the most rapid adsorption has been reported for relatively lower dyes concentrations $[25,33]$.

The pseudo-second-order kinetics model has been widely reported to represent the rate of adsorption for dyes [48, 49] and heavy metals [50] by various adsorbents. The applicability of this model implies that the adsorption is driven by external diffusion at lower concentration wherein the adsorbent-phase mass transfer resistance is dominant, and thereafter the mechanism alternates as chemical-type adsorption through sharing or exchange of electrons between dye molecules and adsorbent at higher concentration [51, 52]. It implies the formation of imaginary bonds due to the electrostatic and dispersive forces interactions. In general, a high adsorbate concentration subsides the solid-phase mass transfer resistance for more adsorbate-adsorbent interactions and adsorption. Notwithstanding that, the vacant active sites progressively decreases with time thus decreasing the rate of adsorption. The rate constant generally decreases with increasing concentration because of the intensified collision of dye molecules $[6,9,15,18,21,24,25$, $39,40,44]$. On the other hand, a greater rate constant with increasing concentration is attributed to a stronger driving force to overcome mass transfer resistance. According to Arrhenius theory, a weak activation energy prompts a high rate constant. Hence, a high rate constant is associated with a rapid adsorption due to less repulsion of molecules and also the contribution of rich surface chemistry [10, 23]. In a related work, the oxidized mesoporous carbon [8] shows a greater rate constant of $71.6 \mathrm{~g} /(\mathrm{mg} \cdot \mathrm{min})$ that is associated with a fast malachite green adsorption with a higher capacity. On the other hand, $\mathrm{Fe}_{3} \mathrm{O}_{4} @ \mathrm{nSiO}_{2} @ \mathrm{mSiO}_{2}$ core-shell microspheres [44] reveals a smaller rate constant of $7.60 \cdot 10^{-5} \mathrm{~g} /(\mathrm{mg} \cdot \mathrm{min})$ despite a superior capacity for congo red. Similarly, zeolite nanostructures from waste aluminium cans [19] demonstrates a rate constant of $6.30 \cdot 10^{-5} \mathrm{~g} /(\mathrm{mg} \cdot \mathrm{min})$ with equilibrium capacity at $227 \mathrm{mg} / \mathrm{g}$.

In this short review, the studies on dyes adsorption by various adsorbents have been reported to be favourable and spontaneous in nature. The thermodynamics of adsorption could be distinguished and discussed from the viewpoint of exothermic or endothermic process. In most literature, the adsorption of dyes is endothermic, in which the adsorption capacity at equilibrium increases with temperature (normally from room temperature to $60{ }^{\circ} \mathrm{C}$ ). This is generally associated with the decrease in liquid viscosity, hence allowing more dye molecules to be entrapped by adsorbent. In certain cases, the increase of solution temperature results in the decrease of dyes removal, rendering an exothermic-type of adsorption. This commonly happens as a result of rapid movement of molecules as temperature increases due to the relatively weak affinity of temperature-sensitive functional groups in the adsorbents [8, 19, 21, 37, 44]. 


\section{Present trends and future outlook}

The pattern of present research on malachite green and congo red adsorption is mainly directed towards developing the novel adsorbents to achieve high removal capacity of dyes. Indeed, the results are overwhelmed in minimizing the research gaps and enriching the body of knowledge. However, several important aspects that worth to be considered are often neglected in much of the published studies. These include: i) cost of adsorbent production, ii) regeneration of adsorbent, iii) adsorption intensity, iv) selectivity, v) kinetics, vi) column adsorption, v) mesoporosity and vi) pore width.

The technical viability for mass production of adsorbent should be evaluated in adsorption studies. In up-scaling the production of adsorbent for industrial applications, an enormous amount of raw material in the magnitude of tons would be needed. In addition, the production in industrial scale with chemicals for modification could be harmful to the environment and may as well escalate the associated costs. In converting the material into adsorbent with special characters, there is always a trade-off between the production and its side implications in compensating the negative consequences towards a sustainable environment. The production cost of adsorbent should be clearly reported and discussed to offer insight into cost-effectiveness for commercialization and practical applications.

The removal of specific dye in a multi-component wastewater is a challenging task because the adsorbent itself may as well take-up several solutes or components at one time. However, the adsorption intensity of adsorbent varies from one solute to another, hence it opens up the room for selective adsorption. For this reason, the affinity profiles towards certain dyes are important to be established, and can be further tailored by adjusting the attributes of surface chemistry and pore texture of adsorbent for the removal of specific dyes. The modification normally involves functionalization of foreign moieties, pore tuning and selection of operating conditions. The adsorption selectivity becomes more economical from the viewpoint of dye recovery and repetitive use of adsorbent in multiple adsorption-desorption cycles. The regeneration of adsorbent for multiple cycles could be sustainable by unlocking the solvents selection for stripping and dye concentration methods.

The adsorption of dyes are relatively slow because dye molecules are big. The time required to attain the equilibrium could be in the order of hours to several days depending on dye concentration. A higher a concentration, a longer would be the equilibrium. Performing the adsorption without knowing the time at which the equilibrium is reached could lead to quasi-equilibrium and misleading interpretation of isotherm. Therefore, cautions should be exercised in carrying out the adsorption kinetics at various concentrations. Moreover, a dedicated research would be needed to speed-up the adsorption of dyes. A slower adsorption, although the capacity at equilibrium is high would jeopardize the performance of adsorption especially in continuous mode.

Dyes are preferred to be adsorbed onto mesoporous adsorbents. Mesopore is defined as the pore in the size range of 2 to $50 \mathrm{~nm}$. The pore width should be big enough to accommodate dye molecules. Generally, the adsorbent with pore size that is about 2.0 to 2.5 times the size of dye molecule is suitable for effective dye adsorption. Often, the authors have stayed silent from discussing the aspects of mesoporosity and pore width of adsorbent for dyes removal. It should be noted, however, this is just a part in the whole interplay of multiple factors governing the adsorption of dyes from water. 


\section{Conclusions}

Studies on the adsorption of dyes have been intensified over the years, with attention at improving the removal capacity by novel adsorbents. Recent progress has demonstrated the development of new adsorbents with excellent traits to remove malachite green and congo red dyes from water. The tremendous values adsorption capacity of $1265 \mathrm{mg} / \mathrm{g}$ for malachite green and $1429 \mathrm{mg} / \mathrm{g}$ for congo red have been reported using oxidized mesoporous carbon and $\mathrm{Fe}_{3} \mathrm{O}_{4} @ \mathrm{nSiO}_{2} @ \mathrm{mSiO}_{2}$, respectively. Clearly, the role of surface chemistry in adsorbent outweighs the influence of surface area for a high adsorption capacity. The synergistic interactions of textural properties, surface functionalization, and operating conditions have been discussed. The adsorption of malachite green and congo red by various adsorbents is favourable and spontaneous, wherein the experimental data could be described by Langmuir and pseudo-second-order kinetics models. Nevertheless, there are several angles in adsorption that worth to be taken into consideration towards process sustainability, such as cost of adsorbent production, regeneration of adsorbent, adsorption selectivity and kinetics, and the role of mesoporosity.

\section{Acknowledgements}

This work is fully supported by Tier1-UTM Research University Grant No. 18H50.

\section{References}

[1] Gautam D, Kumari S, Ram B, Chauhan GS, Chauhan K. A new hemicellulose-based adsorbent for malachite green. J Chem Eng. 2018;6(4):3889-3897. DOI:10.1016/j.jece.2018.05.029.

[2] Sartape AS, Mandhare AM, Jadhav VV, Raut PD, Anuse MA, Kolekar SS. Removal of malachite green dye from aqueous solution with adsorption technique using Limonia acidissima (wood apple) shell as low cost adsorbent. Arab J Chem. 2017;10(2):S3229-S3238. DOI: 10.1016/j.arabjc.2013.12.019.

[3] Wang D, Liu L, Jiang X, Yu J, Chen X. Adsorption and removal of malachite green from aqueous solution using magnetic $\beta$-cyclodextrin-graphene oxide nanocomposites as adsorbents. Colloids Surf A Physicochem Eng Asp. 2015;466:166-173. DOI: 10.1016/j.colsurfa.2014.11.021.

[4] Naseem K, Farooqi ZH, Begum R, Irfan A. Removal of congo red dye from aqueous medium by its catalytic reduction using sodium borohydride in the presence of various inorganic nano-catalysts: A review. J Clean Prod. 2018:187:296-307. DOI: 10.1016/j.jclepro.2018.03.209.

[5] Alver E, Bulut M, Metin AU, Çiftçi H. One step effective removal of congo red in chitosan nanoparticles by encapsulation. Spectrochim Acta A Mol Biomol Spectrosc. 2017;171:132-138. DOI: 10.1016/j.saa.2016.07.046.

[6] Tu NT, Thien TV, Du PD, Chau VT, Mau TX, Khieu DQ. Adsorptive removal of congo red from aqueous solution using zeolitic imidazolate framework-67. J Environ Chem Eng. 2018;6(2):2269-2280. DOI: 10.1016/j.jece.2018.03.031.

[7] Ausavasukhi A, Kampoosaen C, Kengnok O. Adsorption characteristics of congo red on carbonized leonardite. J Clean Prod. 2016;134:506-514. DOI: 10.1016/j.jclepro.2015.10.034.

[8] Zhang X, Lin Q, Luo S, Ruan K, Peng K. Preparation of novel oxidized mesoporous carbon with excellent adsorption performance for removal of malachite green and lead ion. Appl Surf Sci. 2018;442:322-331. DOI: $10.1016 /$ j.apsusc.2018.02.148.

[9] Gupta K, Khatri OP. Reduced graphene oxide as an effective adsorbent for removal of malachite green dye: Plausible adsorption pathways. J Colloid Interface Sci. 2017;501:11-21. DOI: 10.1016/j.jcis.2017.04.035.

[10] Tang S, Zaini MAA. Congo red Removal by $\mathrm{HNO}_{3}$-modified resorcinol-formaldehyde carbon gels. Chem Eng Trans. 2017;56:159-172. DOI: 10.3303/CET1756140.

[11] Leyva-Ramos R. Effect of temperature and $\mathrm{pH}$ on the adsorption of an anionic detergent on activated carbon. J Chem Technol Biotechnol. 1989;45(3):231-240. DOI: 10.1002/jctb.280450308.

[12] Nouri S. Effect of treatment on the adsorption capacity of activated carbon. Adsorpt Sci Technol. 2002;20(9):917-925. DOI: 10.1260/026361703771953578. 
[13] Rajabi M, Mirza B, Mahanpoor K, Mirjalili M, Najafi F, Moradi O, et al. Adsorption of malachite green from aqueous solution by carboxylate group functionalized multi-walled carbon nanotubes: Determination of equilibrium and kinetics parameters. J Ind Eng Chem. 2016;34:130-138. DOI: 10.1016/j.jiec.2015.11.001.

[14] Shah KA, Tali BA. Synthesis of carbon nanotubes by catalytic chemical vapour deposition: A review on carbon sources, catalysts and substrates. Mater Sci Semicon Process. 2016;41:67-82. DOI: 10.1016/j.mssp.2015.08.013.

[15] Tang S, Zaini MAA. Malachite green adsorption by potassium salts-activated carbons derived from textile sludge: Equilibrium, kinetics and thermodynamics studies. Asia-Pac J Chem Eng. 2016;12(1):159-172. DOI: 10.1002/apj.2063.

[16] Vergis BR, Krishna RH, Kottam N, Nagabhushana BM, Sharath R, Darukaprasad B. Removal of malachite green from aqueous solution by magnetic $\mathrm{CuFe}_{2} \mathrm{O}_{4}$ nano-adsorbent synthesized by one pot solution combustion method. J Nanostructure Chem. 2017;8(1):1-12. DOI: 10.1007/s40097-017-0249-y.

[17] Hosseinzadeh H, Ramin S. Fabrication of starch-graft-poly(acrylamide)/graphene oxide/hydroxyapatite nanocomposite hydrogel adsorbent for removal of malachite green dye from aqueous solution. Int $\mathbf{J}$ Biol Macromol. 2018;106:101-115. DOI: 10.1016/j.ijbiomac.2017.07.182.

[18] Dash S, Chaudhuri H, Gupta R, Nair UG. Adsorption study of modified coal fly ash with sulfonic acid as a potential adsorbent for the removal of toxic reactive dyes from aqueous solution: Kinetics and thermodynamics. J Environ Chem Eng. 2018;6(5):5897-5905. DOI: 10.1016/j.jece.2018.05.017.

[19] Abdelrahman EA. Synthesis of zeolite nanostructures from waste aluminum cans for efficient removal of malachite green dye from aqueous media. J Mol Liq. 2018;253:72-82. DOI: 10.1016/j.molliq.2018.01.038.

[20] Altıntıg E, Onaran M, Sarı A, Altundag H, Tüzen M. Preparation, characterization and evaluation of bio-based magnetic activated carbon for effective adsorption of malachite green from aqueous solution. Mater Chem Phys. 2018;220:313-321. DOI: 10.1016/j.matchemphys.2018.05.077.

[21] Baghdadi M, Soltani BA, Nourani M. Malachite green removal from aqueous solutions using fibrous cellulose sulfate prepared from medical cotton waste: Comprehensive batch and column studies. J Ind Eng Chem. 2017;55:128-139. DOI: 10.1016/j.jiec.2017.06.037.

[22] Ghasemi M, Mashhadi S, Asif M, Tyagi I, Agarwal S, Gupta VK. Microwave-assisted synthesis of tetraethylenepentamine functionalized activated carbon with high adsorption capacity for malachite green dye. J Mol Liq. 2016;213:317-325. DOI: 10.1016/j.molliq.2015.09.048.

[23] Tang Y, Zeng Y, Hu T, Zhou Q, Peng Y. Preparation of lignin sulfonate-based mesoporous materials for adsorbing malachite green from aqueous solution. J Environ Chem Eng. 2016;4(3):2900-2910. DOI: 10.1016/j.jece.2016.05.040.

[24] Nekouei F, Kargarzadeh H, Nekouei S, Tyagi I, Agarwal S, Gupta VK. Preparation of nickel hydroxide nanoplates modified activated carbon for malachite green removal from solutions: Kinetic, thermodynamic, isotherm and antibacterial studies. Process Saf Environ. 2016;102:85-97. DOI: 10.1016/j.psep.2016.02.011.

[25] Ghaedi M, Azad FN, Dashtian K, Hajati S, Goudarzi A, Soylak M. Central composite design and genetic algorithm applied for the optimization of ultrasonic-assisted removal of malachite green by $\mathrm{ZnO}$ nanorod-loaded activated carbon. Spectrochim Acta A Mol Biomol Spectrosc. 2016;167:157-164. DOI: 10.1016/j.saa.2016.05.025.

[26] Ghaedi M, Shojaeipour E, Ghaedi A, Sahraei R. Isotherm and kinetics study of malachite green adsorption onto copper nanowires loaded on activated carbon: Artificial neural network modeling and genetic algorithm optimization. Spectrochim Acta A Mol Biomol Spectrosc. 2015;142:135-149. DOI: 10.1016/j.saa.2015.01.086.

[27] Kan Y, Yue Q, Kong J, Gao B, Li Q. The application of activated carbon produced from waste printed circuit boards (PCBs) by H3PO4 and steam activation for the removal of malachite green. Chem Eng J. 2015;260:541-549. DOI: 10.1016/j.cej.2014.09.047.

[28] Shi Z, Xu C, Guan H, Li L, Fan L, Wang Y, et al. Magnetic metal organic frameworks (MOFs) composite for removal of lead and malachite green in wastewater. Colloids Surf A Physicochem Eng Asp. 2018;539:382-390. DOI: 10.1016/j.colsurfa.2017.12.043.

[29] Zhou Y, Ge L, Fan N, Xia M. Adsorption of Congo red from aqueous solution onto shrimp shell powder. Adsorpt Sci Technol. 2018;36(5-6):1310-1330 DOI: 10.1177/0263617418768945.

[30] Yang G, Wu L, Xian Q, Shen F, Wu J, Zhang Y. Removal of congo red and methylene blue from aqueous solutions by vermicompost-derived biochars. Plos One. 2016;11(5):e0154562. DOI: 10.1371/journal.pone.0154562.

[31] Chawla S, Uppal H, Yadav M, Bahadur N, Singh N. Zinc peroxide nanomaterial as an adsorbent for removal of congo red dye from waste water. Ecotoxicol Environ Saf. 2017;135:68-74. DOI: 10.1016/j.ecoenv.2016.09.017. 
[32] Saksornchai E, Kavinchan J, Thongtem S, Thongtem T. Simple wet-chemical synthesis of superparamagnetic CTAB-modified magnetite nanoparticles using as adsorbents for anionic dye Congo red removal. Mater Lett. 2018;213:138-142. DOI: 10.1016/j.matlet.2017.11.015.

[33] Sun P, Chen L, Xu L, Zhu W. Hierarchical porous MgBO $2(\mathrm{OH})$ microspheres: Hydrothermal synthesis, thermal decomposition, and application as adsorbents for congo red removal. Chin J Chem Eng. 2018;26(7):1561-1569. DOI: 10.1016/j.cjche.2018.01.013.

[34] Shojaeipoor F, Elhamifar D, Masoumi B, Elhamifar D, Barazesh B. Ionic liquid based nanoporous organosilica supported propylamine as highly efficient adsorbent for removal of congo red from aqueous solution. Arab J Chem. 2016. DOI: 10.1016/j.arabjc.2016.05.001.

[35] Xu J, Xu D, Zhu B, Cheng B, Jiang C. Adsorptive removal of an anionic dye congo red by flower-like hierarchical magnesium oxide $(\mathrm{MgO})$-graphene oxide composite microspheres. Appl Surf Sci. 2018;435:1136-1142. DOI: 10.1016/j.apsusc.2017.11.232.

[36] Chaukura N, Mamba BB, Mishra SB. Conversion of post-consumer waste polystyrene into a high value adsorbent and its sorptive properties for congo red removal from aqueous solution. J Environ Manage. 2017;193:280-289. DOI: 10.1016/j.jenvman.2017.02.023.

[37] Shaban M, Abukhadra MR, Khan AAP, Jibali BM. Removal of congo red, methylene blue and Cr(VI) ions from water using natural serpentine. J Taiwan Inst Chem Eng. 2018;82:102-116. DOI: /10.1016/j.jtice.2017.10.023.

[38] Tian C, Feng C, Wei M, Wu Y. Enhanced adsorption of anionic toxic contaminant congo red by activated carbon with electropositive amine modification. Chemosphere. 2018;208:476-483. DOI: 10.1016/j.chemosphere.2018.06.005.

[39] Srilakshmi C, Saraf R. Ag-doped hydroxyapatite as efficient adsorbent for removal of congo red dye from aqueous solution: Synthesis, kinetic and equilibrium adsorption isotherm analysis. Micropor Mesopor Mat. 219 (2016), 134-144. DOI: 10.1016/j.micromeso.2015.08.003.

[40] Bharali D, Deka RC. Adsorptive removal of congo red from aqueous solution by sonochemically synthesized NiAl layered double hydroxide. J Environ Chem Eng. 2017;5(2):2056-2067. DOI: 10.1016/j.jece.2017.04.012.

[41] Yang M, Wu Y, Rao R, Wang H. Methanol promoted synthesis of porous hierarchical $\alpha-\mathrm{Ni}(\mathrm{OH})_{2}$ for the removal of congo red. Powder Technol. 2017;320:377-385. DOI: 10.1016/j.powtec.2017.07.074.

[42] Long Y, Yu J, Jiao F, Yang W. Preparation and characterization of MWCNTs/LDHs nanohybrids for removal of congo red from aqueous solution. Trans Nonferrous Met Soc China. 2016;26(10):2701-2710. DOI: 10.1016/s1003-6326(16)64398-4.

[43] Zheng Y, Zhu B, Chen H, You W, Jiang C, Yu J. Hierarchical flower-like nickel(II) oxide microspheres with high adsorption capacity of congo red in water. J Colloid Interface Sci. 2017;504:688-696. DOI: 10.1016/j.jcis.2017.06.014.

[44] Zhang Y, Bai L, Zhou W, Lu R, Gao H, Zhang S. Superior adsorption capacity of $\mathrm{Fe}_{3} \mathrm{O}_{4} @ \mathrm{nSiO}_{2} @ \mathrm{mSiO}_{2}$ core-shell microspheres for removal of congo red from aqueous solution. J Mol Liq. 2016;219:88-94. DOI: 10.1016/j.molliq.2016.02.096.

[45] Lei C, Pi M, Zhou W, Guo Y, Zhang F, Qin J. Synthesis of hierarchical porous flower-like ZnO-AlOOH structures and their applications in adsorption of congo red. Chem Phys Lett. 2017;687:143-151. DOI: 10.1016/j.cplett.2017.09.018.

[46] Satheesh R, Vignesh K, Rajarajan M, Suganthi A, Sreekantan S, Kang M, et al. Removal of congo red from water using quercetin modified $\alpha-\mathrm{Fe}_{2} \mathrm{O}_{3}$ nanoparticles as effective nanoadsorbent. Mater Chem Phys. 2016;180:53-65. DOI: 10.1016/j.matchemphys.2016.05.029.

[47] Tran HN, You SJ, Nguyen TV, Chao HP. Insight into adsorption mechanism of cationic dye onto biosorbents derived from agricultural wastes. Chem Eng Commun. 2017;204(9):1020-1036. DOI: 10.1080/00986445.2017.1336090.

[48] Raval NP, Shah PU, Shah NK. Adsorptive amputation of hazardous azo dye congo red from wastewater: A critical review. Environ Sci Pollut Res. 2016;23:14810-14853. DOI: 10.1007/s11356-016-6970-0.

[49] Raval NP, Shah PU, Shah NK. Malachite green "a cationic dye" and its removal from aqueous solution by adsorption. Appl Water Sci. 2017;7:3407-3445. DOI: 10.1007/s13201-016-0512-2.

[50] Yildiz S. Kinetic and isotherm analysis of $\mathrm{Cu}$ (II) adsorption onto almond shell (Prunus dulcis). Ecol Chem Eng S. 2017;24(1):87-106. DOI: 10.1515/eces-2017-0007.

[51] Zaini MAA, Alias N, Yunus MAC. Bio-polishing sludge adsorbents for dye removal. Polish J Chem Technol. 2016;18 4):15-21. DOI: 10.1515/pjct-2016-0065.

[52] Zaini MAA, Zakaria M, Setapar SHM, Yunus MAC. Sludge-adsorbents from palm oil mill effluent for methylene blue removal. J Environ Chem Eng. 2013;1:1091-1098. DOI: 10.1016/j.jece.2013.08.026. 